\title{
Prompt Fission Gamma-Ray Measurements at UML Research Reactor
}

\author{
Razvan Stanescu ${ }^{1}$, Hadrick Green ${ }^{1}$, Toby Morris ${ }^{1}$, Gencho Rusev ${ }^{2}$, and Marian Jandel ${ }^{3}$ \\ ${ }^{1}$ Physics and Applied Physics PhD candidate, University of Massachusetts-Lowell 01854, USA \\ ${ }^{2}$ Los Alamos National Laboratory, Nuclear and Radiochemistry Group, Los Alamos 87545, USA \\ ${ }^{3}$ Physics and Applied Physics Faculty, University of Massachusetts-Lowell 01854, USA
}

\begin{abstract}
Neutron-induced fission of ${ }^{235} \mathrm{U}$ was studied at the thermal column of the UMass Lowell 1 MW Research Reactor. A collimated, 2.25inch diameter beam of thermal neutrons with the flux of $\sim 5 \times 10^{5} \mathrm{n} / \mathrm{cm}^{2} / \mathrm{sec}$ induced fission reaction on a plate of low-enriched uranium with the areal density $\sim 25 \mathrm{mg} / \mathrm{cm}^{2}$ of ${ }^{235} \mathrm{U}$. We have used the prompt fission-neutron tagging method to identify the fission reaction in the off-line analysis. The method employs the pulse-shape discrimination of neutrons and gamma-ray events in stilbene scintillator and enables identification of coincidence events of prompt fission gamma-rays and prompt fission neutrons in coincidence time intervals less than 20-30 ns. The prompt gamma-ray radiation was detected using two co-linear $\mathrm{NaI}(\mathrm{Tl})$ detectors. The measured spectra of prompt-fission gamma rays between $150 \mathrm{keV}$ and $6 \mathrm{MeV}$ are presented. The results from these initial measurements demonstrate the feasibility of the experimental method. Future measurements with extended arrays of detectors are planned.
\end{abstract}

\section{Introduction}

Prompt fission gamma-ray (PFG) radiation represents approximately 3\% of the total energy released from ${ }^{235} \mathrm{U}$ fission. Understanding of the PFG spectra and multiplicity distribution is critical for the development of future nuclear reactors and impacts the design of their shielding and cooling systems. The main part of the total heating in a non-fuel region of thermal reactors is due to gamma rays (up to 90\%) [Bla07]. The PFG spectrum extends above $10 \mathrm{MeV}$. High energy PFG can penetrate through thick shielding materials. As such, PFG may provide useful signatures for the special nuclear material (SNM) detection, if the SNM is actively interrogated by neutron or high energy gamma-ray probes [Goz09] inducing fission. Studies of the PFG properties are also motivated by basic science to improve models of nuclear fission and statistical evaporation from compound nucleus. The total kinetic energy of fission fragments together with the prompt fission neutron spectra provide important information on the excitation energy of the primary fission fragments (PFF). Neutrons, however, do not carry away significant amount of angular momentum. The PFG, on the other hand, evaporate most of the angular momentum induced in PFF and we expect that the properties of PFG depend on the initial angular momentum distribution of PFF. At high excitation energies, neutron emission dominates PFG emission. However, the 
competition between neutrons and gamma rays is expected near the yrast band, where neutron emission is forbidden because of the high spin.

The previous experimental work on prompt fission gamma includes data taken at Los Alamos [Jan18, Jan14, Jan12] and Budapest [Obe13] on prompt fission gamma spectra (PFGS) from ${ }^{235} \mathrm{U}$ thermal and resonance neutron-induced fission. The existing experimental data are compared to Cascade Gamma-Ray Multiplicity for Fission (CGMF) code simulations [Ste19]. The goal of this work is to establish a technique to measure prompt fission gamma rays with energies up to $20 \mathrm{MeV}$ at the UML Radiation Laboratory, where

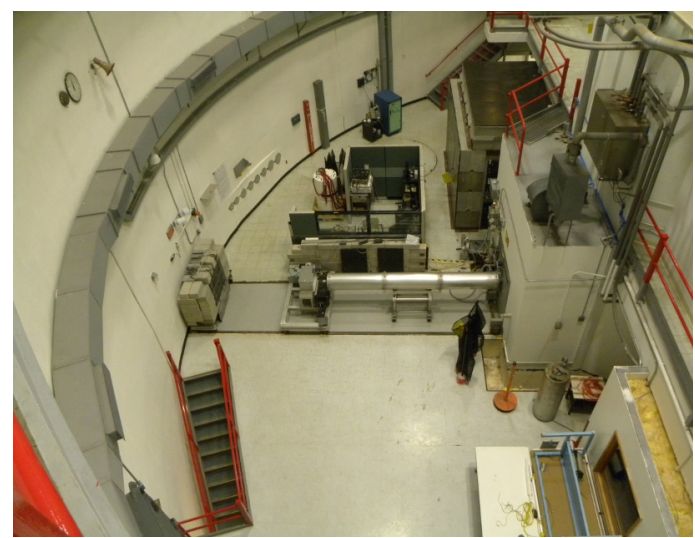

Fig. 1. Thermal column at UML Research Reactor with the removable beam extender.

different sources of neutrons are available and can be measured for thermal, fast and discreet neutron incident energy.

\section{Experiments}

The UMass Lowell Research Reactor (UMLRR) is a pool-type reactor currently licensed to operate at $1 \mathrm{MW}$. The reactor has various experimental facilities that make use of either reactor produced neutrons and gamma rays from high-activity ${ }^{60} \mathrm{Co}$ sources which are colocated in the reactor pool. In-core irradiation facilities provide a fission spectrum of neutrons and gamma rays, with total neutron flux up to $2 \times 10^{13} \mathrm{n} / \mathrm{cm}^{2} / \mathrm{sec}$, and neutron and gamma ray dose rates on the order of $10^{7} \mathrm{rads} /$ hour. Ex-core irradiation facilities include a Fast-Neutron Irradiator that produces predominantly fast neutrons for material testing; a Pneumatic System for instrumental neutron activation analysis; a Thermal Column that provides predominately thermal neutrons for neutron radiography; three Beam Ports that can be used for a variety of sample and detector irradiations; and a Nitrogen-16 Irradiator that is used to calibrate dosimeters to high-energy gamma rays.

Our measurements are carried out at the thermal port of the UMLRR that provides neutrons in the thermal energy range for experimental application. The thermal column is comprised of two separate assemblies. One assembly, a 4 x 4-foot square column, is embedded within the reactor pool biological shield. The other assembly, the thermal column extension, is located between the pool liner and the nuclear core, and is supported by a structural member, which is firmly attached to the pool structure. The center line of the thermal column is aligned with the center line of the reactor core. The $4 \times 4$-foot square embedded column is filled with graphite. A neutron collimator for thermal neutron radiography is located in the center of the column. A lead gamma shield, located on the front end of the thermal column extension, provides shielding of reactor core gammas. $2 \mathrm{MeV}$ fission neutrons travel through 4 feet of graphite and slow to thermal energies. Resulting energies correspond to wave lengths of approximately 0.9 - $6.4 \AA$. A heavy, steel thermal column door is provided as a shield to protect operation personnel against gamma radiation. Four 6" diameter access ports are provided in the face of the door. Each port is fitted with four separate boral-faced plugs. Each plug is drilled and tapped to accommodate a plug removal tool, which is used to insert 


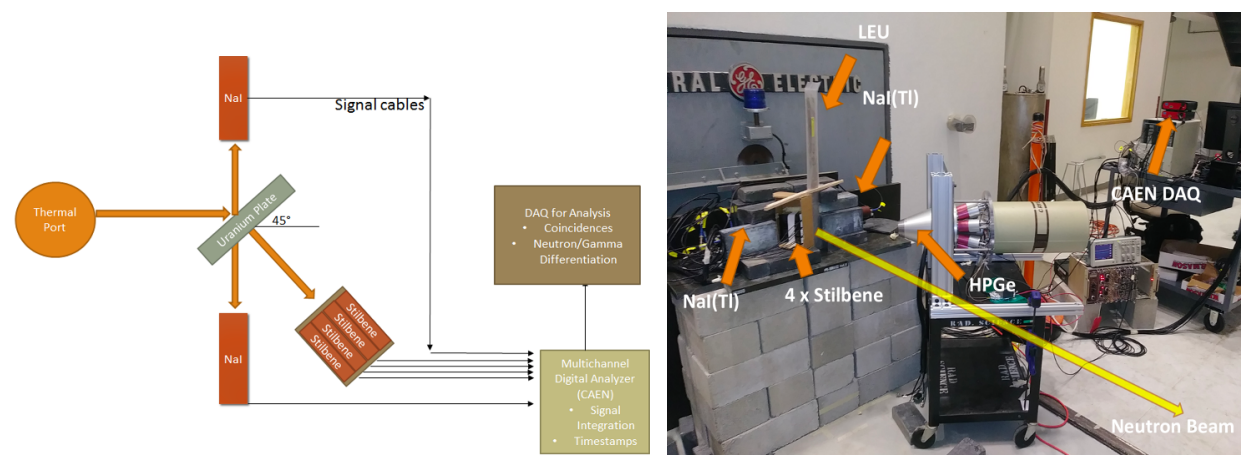

Fig. 2. The physical layout of the fission experiment.

and remove plugs. The maximum thermal neutron flux in the center of the column is $\sim 10^{10}$ $\mathrm{n} / \mathrm{cm}^{2} / \mathrm{sec}$ with a cadmium ratio of $\sim 100$. At the outside face of thermal column (access port), the thermal neutron flux is $\sim 1.5 \times 10^{5} \mathrm{n} / \mathrm{cm}^{2} / \mathrm{sec}$ the cadmium ratio is $\sim 7000$. The neutron flux can be adjusted linearly down to $1.5 \times 10^{5} \mathrm{n} / \mathrm{cm}^{2} / \mathrm{sec}$.

The experimental setup is centered around the collimated 2.5" diameter thermal beam of neutrons from the reactor which is incident on the uranium plate angled at $45^{\circ}$ as shown in Fig. 3 from above. The Low Enriched Uranium (LEU) plate is composed of $\sim 20 \%{ }^{235} \mathrm{U}$ and $\sim 80 \%{ }^{238} \mathrm{U}$. The material is encased in an aluminum housing and measures approximately 24 " x 3" x 0.15 ". The total thickness of ${ }^{235} \mathrm{U}$ in the fuel plate is $\sim 25 \mathrm{mg} / \mathrm{cm}^{2}$. Two NaI(Tl), four stilbene and HPGe detectors are used for the measurements. All detector signals are fed into a CAEN DT5730 digitizer which assigns time stamps and performs signal integration from stilbene and $\mathrm{NaI}(\mathrm{Tl})$ detectors with a 2-ns sampling interval. The DPP-PSD CAEN firmware enables FPGA real-time charge integration and asynchronous data acquisition for all channels.

Gamma rays were measured using two $2 \times 2$ inch $\mathrm{NaI}(\mathrm{Tl})$, placed 2.625 " from the U sample. The $\mathrm{NaI}(\mathrm{Tl})$ crystal has a density of $3.67 \mathrm{~g} / \mathrm{cm}^{3}$ and a decay time of $230 \mathrm{~ns}$. This longer decay time puts a restraint on the maximum count rate in the detector without the pile-up. To counter this, both the distance from the detectors to the target and the reactor power level can be adjusted to control the flux of neutrons and reduce the reaction rates too acceptable level as well as the ambient background rate.

Fast neutrons were detected with stilbene scintillators. Four 1"x1"x4" stilbene detectors are arranged as a 4" x 4" wall placed 4.5" away from the target. Stilbene is an organic scintillator (trans $-\mathrm{C}_{14} \mathrm{H}_{12}$ ) with a density of $1.16 \mathrm{~g} / \mathrm{cm}^{3}$ which scintillates in different decay modes depending on the form of incoming radiation (in our case, neutrons vs gamma). Specifically, three decay components of the scintillation light in the stilbene crystal with the decay constant of $<4 \mathrm{~ns}, \sim 18 \mathrm{~ns},>35 \mathrm{~ns}$ [Zai09] can be categorized into "prompt" and "delayed" fluorescence. A ratio of the prompt and delayed fluorescence in a single event therefore enables incident radiation identification using the pulse shape discrimination (PSD). The ratio differs significantly for different incident radiation. Gamma rays induce significantly more prompt fluorescence compared to neutrons. With the fast response times and a decay time of $4.5 \mathrm{~ns}$ [Leo94], stilbene detectors are ideal for coincidence measurements and are resolvable using a pulse shape discrimination 2-ns resolution CAEN digitizer. In addition, even the slowest component of the fluorescence light guarantees the signals to reach the baseline in a few hundred nanoseconds and enable high counting rate measurements. Because the stilbene-detectors gamma-ray energy resolution is not very good $\sim 18 \%$ (consequence of the small photo-effect cross section in organic crystal), they are not used for spectral intensity 
measurements. Instead, we use them for identifying fission neutrons emitted in coincidence with gamma from higher resolution detectors as a tool for fission reaction tagging [Jan18]. The pulse shape discrimination consists of integrating the event's pulse shape, over time, in two different time intervals from the initial trigger of the pulse - the leading edge. Because of these different integration time lengths, they are referred to as a "short gate" and a "long gate". An appropriate way to visualize the two signal types is by plotting the following ratio compared to the long integral in Fig. 2:

$$
\text { Ratio }=(\text { Long Integral }- \text { Short Integral }) / \text { Long Integral }
$$

The coincident events were built in the off-line analysis to identify the detection of a fission event. In this manner, we were able to gate on the prompt fission neutrons in stilbene and identify coincident prompt fission gamma-rays on an event-by-event basis in the off-line data analysis.

Background measurements were taken in 3 different forms. Firstly, a background radiation measurement of the reactor at power but without the thermal neutron beam passing through. Secondly, a background measurement of the beam passing through the set-up but without the target in place, which allows us to determine the scattering of the radiation through the set-up materials. Finally, considering that the uranium target is encased in an aluminum casing, we used a similarly sized plate of aluminum, or a "dummy" target, to detect the scattering of the radiation off the target. Our results found the latter two background measurements to be nearly identical, and therefore satisfactory for background

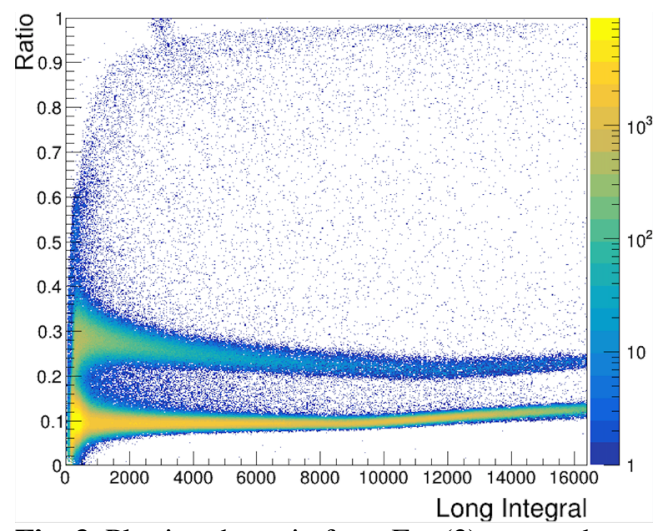

Fig. 3. Plotting the ratio from Eq. (2) versus the long integral of a signal, we can clearly see a delineation between the two radiation types. We can label each signal a neutron or a gamma based subtraction.

\section{Data Analysis}

In the off-line data analysis, the detectors are first synchronized in time when time-offsets are added to each channel to guarantee the true coincidence. This is typically achieved with the ${ }^{60} \mathrm{C}$ source and confirmed with the data taken in beam. Energy calibration of all detectors is carried out as well. The data are then re-analyzed and coincident events are built in a 25ns wide coincidence window. The coincident events that include neutron events from Stilbene detectors are then processed for detail PFG analysis. The width of the window is a variable and can be set to different values and as such, the dependence of PFG on time after the fission event can be studied. Due to the difference in response times of the $\mathrm{NaI}(\mathrm{Tl})$ and stilbene detectors as well as different length cables a very regular difference of timing was found between the two detector types. This consistent difference gives us confidence that the timing process in the digitizer and in our software, is well defined and reflects a replicable difference between the two detector type circuits. Because the signal timing shift was repeatedly found to be $17.5 \mathrm{~ns}$ as seen in Fig. 4, we could adjust the stilbene detectors by this time value so that coincident events are well correlated and we may narrow our coincidence 
window to be smaller around the collective Gaussian distribution and give us more finely sampled coincidence spectra.

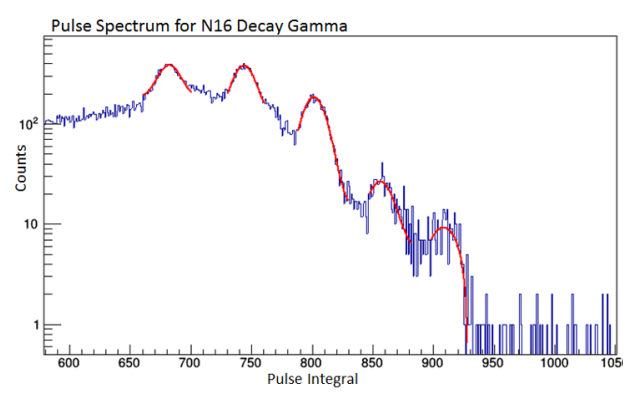

Fig. 4. Pulse spectrum for ${ }^{16} \mathrm{~N}$ decay gamma. The five gamma peaks of a decaying ${ }^{16} \mathrm{~N}$ ranging from 5.1 MeV to 7.1 MeV as detected in a $\mathrm{NaI}$ detector
Energy calibration of all detectors is obtained using the ${ }^{137} \mathrm{Cs},{ }^{60} \mathrm{Co}$, and ${ }^{133} \mathrm{Ba}$ standard calibration sources. Over time however, it was found that the calibrations of $\mathrm{NaI}(\mathrm{Tl})$ would become inaccurate and would drift noticeably between the start and the end of a long day of data taking. We predict this is due either to the well documented variation in response that $\mathrm{NaI}(\mathrm{Tl})$ shows at different temperatures or due to systemic voltage variation in the power sources of the detector high voltage [Ian09]. To mitigate this effect, we found regular calibration before and after hours of data-taking to be sufficient in giving us

accurate spectrum energies. As well, we explored a chi-squared method of comparing spectra of identical set ups with changing energy ranges over time. When comparing subsequent spectra to an original, well calibrated spectrum and scaling the energy domain as needed, the method allowed us to produce accurate calibrations over the course of many individual runs.

We found that at energies above $2 \mathrm{MeV}$, the linear calibrations would begin to deviate in a non-linear way. To appropriately calibrate this region, we used the pool of the reactor as a high energy gamma source. The neutrons coming from the core created ${ }^{16} \mathrm{~N}$ by the following reaction with the oxygen in water: ${ }^{16} \mathrm{O}+\mathrm{n} \rightarrow \mathrm{p}+{ }^{16} \mathrm{~N}$. The ${ }^{16} \mathrm{~N}$ nucleus beta decays with a half-life of $\mathrm{T}_{1 / 2}=7.13$ seconds with the dominant decay gamma rays with energies of 7.115 and $6.129 \mathrm{MeV}$ and probabilities of 0.049 and 0.67 per decay, respectively [Kel93]. Due to the higher rate of pair production at energies over $1 \mathrm{MeV}$ the peaks we expect will be accompanied by its two escape peaks below the main peak. Therefore, we expect to see 6 peaks in total, and due to one overlap we find the 5 peaks in Fig. 4 which we will use in future measurements to calibrate and characterize this non-linear energy region.

\section{Results}

Prompt fission gamma-ray spectrum from thermal neutron induced fission of ${ }^{235} \mathrm{U}$ is shown in Fig 5 by black open squares. The spectrum was obtained by gating on the neutron events detected in stilbene detectors in coincidence with fission gamma rays and coincidence window with of 25 ns. The background spectrum, obtained using the same offline data reduction procedure from the data taken with the $\mathrm{Al}$ dummy in neutron beam are shown in the same Fig 5 by open circles. Both spectra are normalized to 74 minutes of data acquisition. We can see that the background is a few orders of magnitude smaller compared to the prompt fission

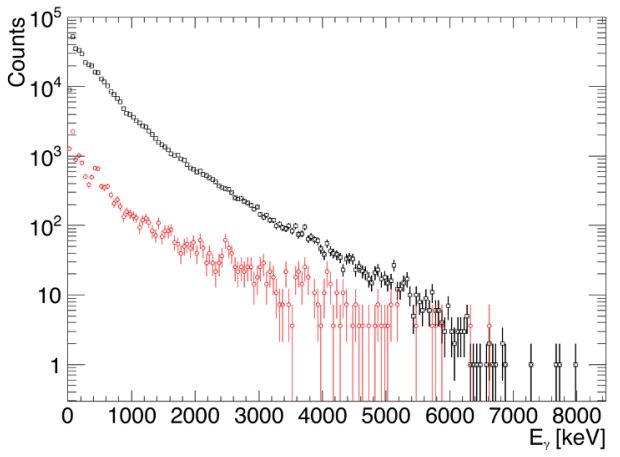

Fig. 5. Prompt fission gamma rays detected from ${ }^{235} \mathrm{U}$ thermal neutron-induced fission in $\sim 2$ hours-long measurement at the UML research reactor operating at $200 \mathrm{~kW}$. The spectrum is obtained by gating on the prompt fission neutron events in stilbene detectors and for the coincidence window width of $25 \mathrm{~ns}$. Background spectrum obtained from measurement with the Al plate is shown using open red squares. 
gamma ray spectrum. This is encouraging for our future efforts to improve the precision and accuracy of the prompt fission gamma measurements.

\section{Discussion and Future Work}

We have successfully designed and carried out measurements of prompt fission gamma-rays in coincidence with the prompt fission neutrons in a collimated beam of thermal neutrons inducing the fission reaction on ${ }^{235} \mathrm{U}$ in a low enriched uranium fuel plate. The neutrons were detected using an array of stilbene scintillators and identified in the off-line pulse shape discrimination analysis. The gamma rays were detected in $\mathrm{NaI}(\mathrm{Tl})$ detectors. Coincident events were built in the offline data analysis with a selectable width of the coincident gate. The results presented in this work are shown for a 25-ns wide coincidence window. In future, we plan to significantly increase the counting statistics and run a week-long measurement in the summer of 2020. To validate our results, detectors of different type, such as $\mathrm{BaF}_{2}$ will be used as well as larger volume $\mathrm{NaI}(\mathrm{Tl})$ detectors. We plan to utilize anti-Compton shields, available at UML, to surround 2-inch diameter detectors and actively reject the scattered gammas from the $\mathrm{NaI}(\mathrm{Tl})$ crystals. This will help improve the response of the detectors after removing the Compton background. Work is under way to field HPGe detectors in the same geometrical configuration and measure high-resolution spectroscopic data for fission gamma rays using the same fission neutron tagging method. Finally, the Geant 4 and MCNP simulations will be carried out for our experimental set-up to better understand the scattering background and response function of the detectors. We would like to thank the staff of UML Research Reactor and Radiation Safety Office, for providing hours of reactor operations and support to field and run the experiment. We are indebted to Nuclear Radiochemistry Group at LANL for stilbene detectors of NEUANCE [Jan18] that were used in this measurement.

\section{References}

[Bla07] D. Blanchet et al., Annals of Nucl. Energy 35 (2007) 731-745.

[Goz09] T. Gozani, IEEE Transactions on Nucl. Sc., 56, 736 (2009).

[Jan18] M. Jandel et al., Correlated fission data measurements with DANCE and NEUANCE, Nuclear Instruments and Methods in Physics Research Section A: Accelerators, Spectrometers, Detectors and Associated Equipment, Volume 882 (2018) 105-113.

[Jan14] M. Jandel et al., Physics Procedia 59 (2014) 101-106.

[Jan12] M. Jandel et al., Los Alamos Report No. LA-UR-12-24975 (2012), to be published.

[Obe13] A. Oberstedt, T. Belgya, R. Billnert et al., Phys. Rev. C 87, 051602 (2013).

[Ste19] I. Stetcu. Evaluation of the Prompt Fission Gamma Properties for Neutron Induced Fission of ${ }^{235,238} \mathrm{U}$ and ${ }^{239} \mathrm{Pu}$. (2019).

[Zai09] N. Zaitseva, et. al. Neutron detection with single crystal organic scintillators, SPIE Hard X-Ray, Gamma-Ray, and Neutron Detector Physics, 11 (2009).

[Leo94] W. Leo, Techniques for Nuclear and Particle Physics Experiments, A How-To Approach, Springer Science \& Business Media, 159-164 (1994)

[Kel93] J. H. Kelley, D. R. Tilley, H. R. Weller and C. M. Cheves, NP 5641 (1993). Data extracted using the NNDC On-line Data Service from the ENSDF database, revision of December, 1993.

[Ian09] K. D. Ianakiev, B. S. Alexandrov, P. B. Littlewood, and M. C. Browne, Nuclear Instruments and Methods in Physics Research Section A: Accelerators, Spectrometers, Detectors and Associated Equipment, Volume 607, Issue 211, Pages 432-438 (2009), 